\title{
Turbulent Transport in Premixed Flames Approaching Extinction
}

\author{
K. H. H. Goh, P. Geipel, R. P. Lindstedt* \\ Department of Mechanical Engineering, Imperial College, London SW7 2AZ, UK \\ *Corresponding author. \\ Fax: +44 207594 5696. Email: p.lindstedt@imperial.ac.uk \\ Colloquium: TURBULENT FLAMES
}

Total length is 6195 words, determined by method 2 for LaTeX users.

Main text: 3820; References: 508;

Eq. (1): 18 (method 2); Eq. (2): 22 (method 2); Eq. (3): 11 (method 2);

Eq. (4): 37 (method 2); Eq. (5): 29 (method 2)

Table 1: 106 (method 2)

Table 2: 156 (method 2)

Figure 1: 154 (method 2)

Figure 2: 194 (method 2)

Figure 3: 99 (method 2)

Figure 4: 154 (method 2)

Figure 5: 154 (method 2)

Figure 6: $154(\operatorname{method} 2)$

Figure 7: 154 (method 2)

Figure 8: 154 (method 2)

Figure 9: 99 (method 2)

Figure 10: 172 (method 2)

Supplementary data is provided with this paper. 


\title{
Turbulent Transport in Premixed Flames Approaching Extinction
}

\author{
K. H. H. Goh ${ }^{\mathrm{a}}$, P. Geipel ${ }^{\mathrm{b}}$, R. P. Lindstedt* \\ Department of Mechanical Engineering, Imperial College, London, SW7 2AZ, UK
}

\begin{abstract}
The turbulent transport in premixed flames approaching extinction has been characterised in terms of statistical properties using an opposed jet burner featuring fractal grid generated turbulence. The burner was used in a symmetric twin flame configuration featuring pre-vaporised cyclopentane and JP-10 (exotetrahydrodicyclopentadiene) at equivalence ratios of 0.75 and 0.85 . The choice of fuels follows from the practical importance of JP-10 as an aviation fuel with cyclic $\mathrm{C}_{5}$ compounds appearing as breakdown products. The bulk velocity was set to $3.0 \mathrm{~m} / \mathrm{s}$ resulting in a turbulent Reynolds number of 120 . The obtained data includes conditional velocity statistics, flame curvature information and scalar fluxes. The conversion between conventionally and Favre averaged statistics follows the Bray-Moss-Libby theory and the assumption that finite reaction interface thickness effects can be neglected. The impact of deviations from the latter on statistics is explored and results suggest that the effect is modest for interface thicknesses less than $20 \%$ of the turbulent flame brush. The experimental data obtained is sufficient to enable terms up to and including triple correlations to be evaluated in closed form. The work also clearly illustrates the rapid transition from nongradient to gradient turbulent transport as the extinction limit is approached.
\end{abstract}

Keywords: Fractal grids, Premixed, BML, Opposed jets, Liquid fuels

${ }^{*}$ Corresponding author. Fax: +44 2075945696.

Email address: p.lindstedt@imperial.ac.uk (R. P. Lindstedt)

Current address: ${ }^{a}$ Institute of Materials Research and Engineering 3, Research Link, Singapore 117602.

${ }^{\mathrm{b}}$ Siemens Industrial Turbomachinery AB, SE-612 83 Finspong, Sweden. 


\section{Introduction}

Bilger et al. [1] observe that turbulent premixed combustion is commonly agreed to be inherently complex with increasingly wide application in internal combustion engines, modern gas turbines for power generation and jet engine afterburners [2]. The increased use stems from a desire to reduce emissions while obtaining higher efficiencies. Bray, Moss and Libby [3] derived the arguably most successful framework (BML) for analysing turbulent premixed combustion on the basis of a statistical description and Bray [4] presented a recent review of the approach. The current contribution considers statistically stationary flames in the context of this framework through experimental measurements using a twin flame opposed jet geometry featuring fractal grid generated turbulence. The density segregation technique derived by Goh et al. [5] is used to detect flame iso-contours and to obtain conditional statistics. Opposed jet flames have been used extensively in the past (e.g. [6-10]) and recent contributions include those of Geipel et al. [11], Goh et al. [12, 13] and Coriton et al. [14].

The use of fractal grids to generate multiscale turbulence was proposed by Vassilicos and Hunt [15] and subsequently investigated by Hurst and Vassilicos [16] and Stresing et al. [17]. Fractal grids have shown significant promise in generating elevated levels of turbulence while maintaining flows free of bulk instabilities. The ideal grid for the opposed jet configuration was identified by Geipel et al. [11] and found to produce a 100\% increase in Reynolds stresses compared to traditional grids with $4 \mathrm{~mm}$ holes as used previously by Böhm et al. [18]. Comprehensive statistical information for twin methane and propane flames has also been obtained by Goh et al. [13] using synchro- 
nised velocity and scalar statistics to provide conditional dissipation and flow field structure information via conditional Proper Orthogonal Decomposition (CPOD). A revised configuration was used by Goh et al. [12] to study the transition from conventional premixed turbulent JP-10 flames to a Homogeneous Charge Diffusion Ignition (HCDI) [19] related flameless oxidation mode using single JP-10 flames. It was observed that conventional flames ceased to exist after transition to flameless oxidation, with reaction interfaces residing on the instantaneous stagnation plane, similar to flames close to extinction as observed by Kostiuk et al. [6]. Furthermore, a shift from non-gradient to gradient turbulent transport was observed for flames beyond the normal extinction point [12] as the combustion mode shifted to flameless oxidation. Under such conditions, reactant conversion must be supported by hot combustion products emerging from one of the nozzles.

By contrast, the current study considers turbulent transport in flames approaching extinction in a conventional back-to-back twin flame configuration that can more readily be related to conventional premixed turbulent flame theories (e.g. BML). Density segregation methods e.g. [5, 12, 20], used in the current work, result in flame isocontours one pixel in width. For flames with a broader reaction interface, the probability of detecting a burning mixture may become significant. The effect has been assessed experimentally through comparisons with OH-PLIF $[12,21]$ and the current work provides a further theoretical analysis of the impact on statistics. The approach is based on a stochastic analysis of the impact of the ratio of the flame thickness $\left(\delta_{f}\right)$ or, more generally, the instantaneous interface thickness $\left(\delta_{I}\right)$ to the turbulent reaction zone (flame brush) thickness $\left(\delta_{t}\right)$. The analysis supports the 
conclusion [12] that the impact on reaction progress variable $(c)$ statistics is negligible under the current experimental conditions. The finding allows direct comparisons of experimental results with theoretical investigations of premixed turbulent flames stabilised in evolving multiscale turbulence. Finally, a set of equations [22] that enable the closed form conversion between conventionally and Favre averaged terms, up to and including triple correlations, on the basis of presented experimental data are reported.

\section{Experimental Techniques}

The turbulent opposed jet configuration corresponds to that of Goh et al. [12] with the exception that the optimal fractal grids identified by Geipel et al. [11] were used in both nozzles in order to produce a twin flame configuration. Impact plates were placed upstream of the fractal grids in order to isolate upstream conditions from grid generated turbulence [23] and hence to simplify the boundary conditions leading to a reduced size of the physical domain required for computational studies. The nozzle exits were one nozzle diameter D (= $30 \mathrm{~mm})$ apart. Twin premixed flames of cyclopentane and JP-10 were investigated using equivalence ratios $(\phi)$ of 0.75 and 0.85 . Flow rates were such that mixtures from each nozzle had a bulk velocity $\left(U_{b}\right)$ of $3.0 \mathrm{~m} / \mathrm{s}$ (at $298 \mathrm{~K}$ ). Cyclopentane and JP-10 were heated to $353 \mathrm{~K}$ and $473 \mathrm{~K}$, respectively, with nozzle exit temperatures of $333 \mathrm{~K}$ and $408 \mathrm{~K}$ maintained in order to prevent fuel recondensation.

The flow control system was the same as used by Goh et al. [12] with uncertainties in flow rates $\leq 0.8 \%$ for each fluid. Flow rates of cyclopentane and JP-10 were metered and vapourised using Bronkhorst CoriFlow M53 and 
CEM W-303A units with the vapourised fuel stream mixed with air and split equally using two needle valves. Measurements performed using GCMS on the reactant stream using an Agilent 7890A series GC with a 5975C inert MSD/DS Turbo EI Bundle equipped with 60 m DB-1 column have confirmed that no cracking of the fuel takes place. Coflow velocities were set to $0.3 \mathrm{~m} / \mathrm{s}$ to remove any large scale bulk motion. The reactant streams were mixed further with seeded air within heated hoses, temperature controlled with an accuracy of $\pm 1 \mathrm{~K}$, for about $3 \mathrm{~m}$ before being introduced into the nozzles. A digital camera was used to capture the $\mathrm{CH}$ chemiluminescence from both flames and measurements performed only when $\mathrm{CH}$ intensities were equal.

Particle Image Velocity (PIV) measurements were conducted in 2D using a $120 \mathrm{~mJ}$ Solo-New Wave Nd:YAG laser. Both the upper and lower streams were seeded with aluminium oxide particles of about $3 \mu \mathrm{m}$ diameter with correlation between Mie scattered images calculated using decreasing interrogation window sizes from $128 \times 128$ via $64 \times 64$ to $32 \times 32$ with a $50 \%$ overlap resulting in velocity vectors spaced about $0.4 \mathrm{~mm}$ apart. Laser pulses were separated by $40 \mu$ s to minimise spurious vectors, and 1000 image pairs were obtained at around $5 \mathrm{~Hz}$ for each case (e.g. [8, 11, 12]).

\section{Postprocessing techniques}

For every image pair the density segregation method of Goh et al. [5] was used to detect flame isocontours in order to synchronise the instantaneous reaction progress variable $(c)$ with velocity vectors. The method has been shown to be accurate to the order of the reaction interface thickness $\left(\delta_{I}\right)[5]$ and used by Goh et al. $[12,13,23]$ to obtain synchronised velocity- 
scalar statistics. The density segregation method bypasses the need for an additional laser for flame front detection and overcomes problems associated with laser alignment. One drawback is that detected isocontours (reaction interfaces) are one pixel wide $(\sim 0.026 \mathrm{~mm})$. Reaction interfaces are often thicker, as shown by Goh et al. [12], and care needs to be taken to ensure applicability of the method. The flames investigated here are directly related to the latter study where the use of OH-PLIF via the method of Kerl et al. [21] confirmed the applicability. However, the matter has also been investigated theoretically as outlined below.

Goh [22] developed an approach to ascertain the impact of a finite mean reaction layer (flame) thickness $\left(\delta_{f}\right)$ on measured flow field statistics using a stochastic mapping method. It was shown that the effects of a finite interface thickness on profiles of the mean progress variable were insignificant even when it reached $20 \%$ of the turbulent flame brush thickness $\left(\delta_{t}\right)$. The simulated flame brush was defined using a Gaussian $p d f$ for flame location, such that the distance between the 5th and 95th percentiles of its cumulative distribution function $(c d f)$ was equal to the measured value. Profiles of instantaneous reaction sheets were also assumed to be in the shape of a Gaussian $c d f$ with the distance between the 5th and 95th percentiles equal to the respective measured values of $\delta_{f}\left(\right.$ or $\left.\delta_{I}\right)$. A random number generator [24] was used to simulate the locations of instantaneous flame isocontours using the Gaussian $p d f$ of the flame location and instantaneous profiles were mapped onto the locations to obtain the first and second moments of the 'true' reaction progress variable. The corresponding profiles assuming that flame sheets were infinitesimally thin $\left(\delta_{f} \rightarrow 0\right)$ were also obtained. First and 
second moments of the 'detected' reaction progress variable were obtained using a method accurate to the order of $\delta_{f}$. The detected isocontours were assumed to be uniformly distributed between the 0.5 th and 99.5th percentiles of the Gaussian $c d f$ for the 'true' instantaneous flame. The infinitesimally thin isocontours were mapped relative to the true signal in order to obtain 'detected' first and second moments of the reaction progress variable. The same technique was applied here to ascertain the impact on the first and second moments of progress variable statistics. The former method was used to show the relative effects on progress variable statistics if the flames were assumed to be infinitesimally thin, while the latter method included the additional effects of uncertainties in the detection of flame isocontours.

To measure the instantaneous flame interface thickness a purpose written algorithm was used with a Savitzky-Golay filter [25] to fit second order polynomials to the detected flame isocontours by using 10 and 15 neighbouring points $\left(n_{f i t}\right)$ in each direction, with sample images shown in Fig. 1. The same fitting algorithm was also used with $n_{\text {fit }}$ set to 5 to ascertain the impact on results. The resulting mean absolute deviations (comparing $n_{f i t}=5$ and 15) in locations of fitted isocontours $(\overline{|\Delta d|} \sim 0.003 \mathrm{~mm})$ and direction of normal vectors to the isocontours $(\overline{|\Delta \theta|} \sim 2.5$ degrees) showed that the location and orientation of the fitted isocontours were relatively insensitive to the fitting parameters. Subsequently, bilinear interpolation was used to map profiles of PIV image intensities normal to the flame for all points on the isocontours for each set of 1000 image pairs. The profiles for each image were normalised using the mean intensity in the reference windows used for density segregation as outlined by Goh et al. [12]. The mean profiles of normalised PIV 
image intensities were obtained using detected flame isocontours for each set of measurements and the mean flame interface thickness was obtained for each set of measurements by fitting error functions to the profiles. The magnitude of $\delta_{f}$ was defined as the distance between the 5th and 95th percentiles of the error functions. The flame brush thickness $\left(\delta_{t}\right)$ was also obtained in the manner of Goh et al. [12]. Values of curvature $h$ (positive when concave to product stream) were obtained using the above method.

\section{Results and Discussion}

The obtained mean interface $\left(\delta_{f}\right)$ and flame brush $\left(\delta_{t}\right)$ thicknesses are presented in Table 1. The latter were in the range $7.5 \leq \delta_{t}(\mathrm{~mm}) \leq 8.5$ and hot wire measurements in the corresponding isothermal flow produced integral length scales $\left(L_{I}\right)$ of around $4.0 \mathrm{~mm}[22]$. Accordingly, the flame brush is approximately twice the integral length scale in the reactant flow. Values of the flame (reaction layer) thickness $\delta_{f}$ showed a modest decrease as the equivalence ratio was increased from 0.75 to 0.85 , similar to the measurements by Goh et al. [12], where the thickness decreased from about 0.78 to $0.58 \mathrm{~mm}$ as the equivalence ratio increased from 0.60 to 0.80 . The flame thicknesses were similar for both JP-10 flames, probably influenced by a slight asymmetry at the equivalence ratio of 0.75 . The error estimation technique outlined above was applied using the determined values of $\delta_{f}$ and $\delta_{t}$ with the detected flame isocontours randomly displaced from the true isocontours. The resulting normalised errors in the turbulent flame thickness $\left(\delta_{t}\right)$, which is directly related to the reaction progress variable $(c)$, were found to be around 0.44 - $0.86 \%\left(\Delta \delta_{t} / \delta_{t}\right)$. The highest $\delta_{f} / \delta_{t}$ in the current context was around $12 \%$ 
for the JP-10 flame with $\phi=0.75$. Even when the detection of flame isocontours had uncertainties of the order of $\delta_{f}$, the expected deviations in $\bar{c}$ were negligible and profiles of $\overline{c^{\prime} c^{\prime}}$ showed that true peak values were around 0.22 compared to measured values of 0.25 with minimal deviations in the profiles as shown in Fig. 2. Hence, the impact of a finite flame (reaction layer) thickness on statistics is not significant in the current work.

It is not possible to present all the obtained statistical information and the focus is placed on data obtained along the burner centreline, where $\bar{V}, \bar{V}_{r}, \bar{V}_{p}$, and $\overline{v^{\prime} c^{\prime}}$ are zero. Measurements presented include unconditional and conditional axial terms $\bar{U}, \bar{U}_{r}, \bar{U}_{p}, \overline{u^{\prime} u^{\prime}}, \overline{u_{r}^{\prime} u_{r}^{\prime}}, \overline{u_{p}^{\prime} u_{p}^{\prime}}$ and $\overline{u^{\prime} c^{\prime}}$, the corresponding radial terms $\overline{v^{\prime} v^{\prime}}, \overline{v_{r}^{\prime} v_{r}^{\prime}}$ and $\overline{v_{p}^{\prime} v_{p}^{\prime}}$, as well as the first two moments of the reaction progress variable $\bar{c}$ and $\overline{c^{\prime} c^{\prime}}$. (The data enables terms up to and including triple correlations to be evaluated in closed form [22]). The conversion between conventionally (e.g. $\bar{c}$ ) and Favre (e.g. $\widetilde{c}$ ) averaged statistics, more commonly used in model formulations, also follows. The expansion ratio $\left(\tau=\frac{\rho_{r}}{\rho_{p}}-1\right)$, readily estimated (e.g. via laminar flame computation), is the only unclosed term. Equivalent equations have been derived by Bray et al. [3] for some of the correlations. The Favre averaged axial scalar flux can be evaluated by re-arranging Eq. (2).

$$
\begin{aligned}
\widetilde{c} & =\frac{\bar{c}}{1+\tau-\tau \bar{c}} \\
\overline{u^{\prime} c^{\prime}} & =\widetilde{u^{\prime \prime} c^{\prime \prime}} \frac{1+\tau}{(1+\tau \widetilde{c})^{2}}
\end{aligned}
$$

These equations only hold for infinitesimally thin flames. The corresponding triple correlation can be obtained from Eq. (4), 


$$
\begin{aligned}
\widetilde{U}= & (1-\widetilde{c}) \bar{U}_{r}+\widetilde{c} \bar{U}_{p} \\
\widetilde{u " u^{\prime c} "}= & \widetilde{c}(1-\widetilde{c})\left[\bar{U}_{p}^{2}-\bar{U}_{r}^{2}+2 \widetilde{U}\left(\bar{U}_{r}-\bar{U}_{p}\right)\right. \\
& \left.+\left(\overline{u_{p}^{\prime} u_{p}^{\prime}}-\overline{u_{r}^{\prime} u_{r}^{\prime}}\right)\right]
\end{aligned}
$$

and, finally, the axial Reynolds stress follows from a re-arrangement of Eq. (5). The corresponding equations for radial components follow by similarity and the general expressions are listed in the Supplemental material.

$$
\overline{u^{\prime} u^{\prime}}=\widetilde{u " u^{\prime \prime}}+\widetilde{u " u^{\prime \prime} c^{\prime \prime}} \frac{\tau}{(1+\tau \widetilde{c})}-\left(\frac{\tau \widetilde{u^{\prime c} c^{\prime \prime}}}{1+\tau \widetilde{c}}\right)^{2}
$$

Boundary conditions in terms of mean velocity components and Reynolds stresses at a distance of $2 \mathrm{~mm}$ from each nozzle exit are included in the Supplemental material in order to aid computational studies. Centreline profiles of mean axial velocities are shown in Fig. 3, where deviations from straight line profiles (isothermal) increased with equivalence ratios, similar to previous findings by Kostiuk et al. [6]. The corresponding unconditional Reynolds stresses are shown in Fig. 4, where peak radial components at the nominal stagnation plane increased with equivalence ratio (cf. Goh et al. [13]). Reaction progress variable statistics, see Fig. 5, show that flames move further from the nominal stagnation plane as the equivalence ratio is increased (cf. Goh et al. [12]). Conditional velocities are shown in Fig. 6, where the reactant velocities decreased slightly and product velocities increased as the equivalence ratio was increased. Conditional axial reactant and product Reynolds 
stresses are shown in Fig. 7. The corresponding profiles for the radial components, see Fig. 8, show that the reactant Reynolds stresses were almost constant, while product stresses increased towards the nominal stagnation plane, with slightly higher values as the equivalence ratio increased. For JP10 flames at an equivalence ratio of 0.75 , radial reactant Reynolds stresses showed a peak before the stagnation plane, possibly due to slightly different equivalence ratios between the upper and lower flames. Results for scalar fluxes, see Fig. 9, show large uncertainties for this case. The proximity to global extinction ( $\phi_{\text {ext }} \sim 0.7$ for both fuels) coupled with the transition in flame propagation mode are likely contributors. However, it can be observed that as the equivalence ratio is increased, there is a transition from gradient to counter gradient transport and that the same trend is obtained for both fuels. It is imperative that computational models reflect such phenomena accurately as the propagation mode impacts flame dynamics and the stabilisation point. Furthermore, results show that flame extinction in the current twin flame configuration and the transition to flameless oxidation with combustion stabilised against a hot product stream [12] are both accompanied by a transition to gradient transport. The mode of transport is affected by a number of factors, including the expansion ratio. Lindstedt and Váos [26] derived a relationship based on the balance between mean strain (production term tensor) and mean pressure gradient effects. However, in the turbulent opposed jet configuration the geometry imposed pressure gradient exerts a significant influence on the transition. Efforts have been made to account for such effects [27], though a comprehensive evaluation of the resulting model formulations present difficulties in the current context, while Chen and Bil- 
ger [28] analysed the transition using Bunsen flames.

Curvature statistics for the reaction interfaces was obtained using the Savitzky-Golay filter [25] based technique outlined above and with three values of $n_{f i t}(=5,10,15)$ to obtain fitted flame isocontours. By using a range of values, it was possible to ascertain the relative sensitivity of derived curvatures to the fitting parameter as given in Table 2 . It can be noted that discrepancies are notably decreased as $n_{f i t}$ is raised from 5 to 10 . The obtained $p d f$ of curvature is shown in Fig. 10 and, again, it can be observed that for the two higher values there is good agreement apart from at zero curvature. The latter discrepancy is very strong when $n_{f i t}$ is set to 5 and a plausible explanation is that too little local information was available to obtain accurate values for large local radii corresponding to the peak at zero curvature. Also, as the fundamental definition of curvature $\left(h(s)=\left(x^{\prime} y^{\prime \prime}-x^{\prime \prime} y^{\prime}\right) /\left(x^{\prime 2}+y^{\prime 2}\right)^{3 / 2}\right)$ involves first and second order gradients, the uncertainties are expected to be large. Furthermore, the 'optimum' fitting parameter is arguably dependent on the local spectral content as isocontours are a complex superposition of multiple scales. Accordingly, an accurate determination of curvature statistics may require prior knowledge of the geometrical nature of the isocontours. Nevertheless, the effects of equivalence ratio on curvature statistics is modest even close to the global extinction limit. The latter is consistent with related experimental studies for stable flames. Bradley et al. [29] analysed a wide range of flames and showed the applicability of a symmetric Gaussian form of the $p d f$ of curvature with the peak value at zero curvature linearly related to the Damköhler number as $D a^{1 / 2}$. Yuen and Gülder [30] also showed a symmetric Gaussian form of the $p d f$ for methane and propane flames, while 
Gashi et al. [31] addressed comparisons between 2D slices obtained experimentally and DNS studies. Sadanandan et al. [32] showed that a broadly Gaussian $p d f$, subject to local conditions, was obtained for a range of stable syngas flames operating on a swirl burner at pressures up to 20 bar. Here, the suggestion by Bradley et al. [29], see Supplemental material, results in a peak value of 0.73 and a somewhat narrower distribution (see Fig. 10). The current study thus suggests that a (near) Gaussian distribution extends to flames undergoing a flame propagation mode transition close to global extinction.

\section{Conclusion}

The turbulent transport in premixed flames of JP-10 and cyclopentane approaching extinction has been characterised in terms of statistical properties using a twin flame opposed jet burner configuration featuring fractal grid generated turbulence. A comprehensive set of statistical information has been provided along the axis of the burner to support comprehensive comparisons with computational studies. The obtained data includes conditional velocity statistics, flame curvature information and scalar fluxes and permits the closed form conversion between conventionally and Favre averaged statistics on the assumption that finite reaction interface thickness effects can be neglected. It has been shown that the impact of the latter on measured statistics is not significant in the current work. Results also clearly illustrate the rapid transition from non-gradient to gradient turbulent transport as the extinction limit is approached. The transition in flame propagation mode is important as it affects flame dynamics and the flame stabilisation 
point. The results also confirm that a (near) Gaussian $p d f$ of flame curvature prevails close to global extinction and that the change in flame propagation mode is related to the disappearance of the conventional premixed turbulent flame with a transition to a flameless oxidation mode required to support combustion in leaner mixtures.

\section{Acknowledgements}

The research was carried out with the financial support of the Office of Naval Research Global under grant N62909-12-1-7127 and European Office of Aerospace Research and Development/Air Force Office of Scientific Research under grant FA8655-13-1-3024. The support of Dr Gabriel Roy, Dr Chiping Li and Dr Gregg Abate is gratefully acknowledged. The authors would also like to thank Mr Fabian Hampp for assisting with the experiments.

\section{References}

[1] R.W. Bilger, S.B. Pope, K.N.C. Bray, J.F. Driscoll, Proc. Combust. Inst. 30 (2005) 21-42.

[2] R.W. Bilger, Prog. Energ. Combust. 26 (4) (2000) 367-380.

[3] K.N.C. Bray, P.A. Libby, J.B. Moss, Combust. Flame 61 (1) (1985) $87-102$.

[4] K.N.C. Bray, Laminar Flamelets and the Bray, Moss and Libby Model, in Turbulent Premixed Flames, Eds. N. Swaminathan and K.N.C. Bray, Cambridge University Press, ISBN 978-0-521-76961-7, Cambridge, UK, pp. 41-60 (2011). 
[5] K.H.H. Goh, P. Geipel, R.P. Lindstedt, Conditional Statistics of Lean Premixed Turbulent Opposed Jet Flames Using Particle Image Velocimetry and Density Segregation, in: Proceedings of the European Combustion Meeting, 2011, p. 1.

[6] L.W. Kostiuk, K.N.C. Bray, R.K. Cheng, Combust. Flame 92 (4) (1993) 396-409.

[7] K. Sardi, A.M.K.P. Taylor, J.H. Whitelaw, Combust. Flame 120 (3) (2000) 265-284..

[8] R.P. Lindstedt, D.S. Luff, J.H. Whitelaw, Proc. Combust. Inst. 31 (1) (2007) 1459-1466.

[9] D. Geyer, A. Kempf, A. Dreizler, J. Janicka, Combust. Flame 143 (4) (2005) 524-548.

[10] B. Böhm, C. Heeger, I. Boxx, W. Meier, A. Dreizler, Proc. Combust. Inst. 32 (2) (2009) 1647-1654.

[11] P. Geipel, K.H.H. Goh, R.P. Lindstedt, Flow Turbul. Combust. 85 (3) (2010) 397-419.

[12] K.H.H. Goh, P. Geipel, F. Hampp, R.P. Lindstedt, Proc. Combust. Inst. 34 (2) (2013) 3311-3318.

[13] K.H.H. Goh, P. Geipel, F. Hampp, R.P. Lindstedt, Fluid Dyn. Res. 45 (6) (2013) 061403.

[14] B. Coriton, J.H. Frank, A. Gomez, Combust. Flame 160 (11) (2013) 2442-2456. 
[15] J.C. Vassilicos, J.C.R. Hunt, Proc. Roy. Soc. A-Math Phys. 435 (1895) (1991) 505-534.

[16] D. Hurst, J.C. Vassilicos, Phys. Fluids 19 (2007) 1-31.

[17] R. Stresing, J. Peinke, R.E. Seoud, J.C. Vassilicos, Phys, Rev. Lett. 104 (19) (2010) 194501.

[18] B. Böhm, D. Geyer, A. Dreizler, K.K. Venkatesan, N.M. Laurendeau, M. W. Renfro, Proc. Combust. Inst. 31 (1) (2007) 709-717.

[19] M. de Joannon, A. Matarazzo, P. Sabia, A. Cavaliere, Proc. Combust. Inst. 31 (2007) 3409-3416.

[20] A.M. Steinberg, J.F. Driscoll, S.L. Ceccio, Exp. Fluids 44 (2008) 985999.

[21] J. Kerl, T. Sponfeldner, F. Beyrau, Combust. Flame 158 (10) (2011) 1905-1907.

[22] K.H.H. Goh, Investigation of Conditional Statistics in Premixed Combustion and the Transition to Flameless Oxidation in Turbulent Opposed Jets, PhD thesis, Imperial College London, United Kingdom, 2013.

[23] K.H.H. Goh, P. Geipel, R.P. Lindstedt, Combust. Flame. (In Press) http://dx.doi.org/10.1016/j.combustflame.2014.03.010

[24] MATLAB and Statistics Toolbox Release 2012b, The MathWorks, Inc., Natick, Massachusetts, United States.

[25] A. Savitzky, M.J.E. Golay, Anal. Chem. 36 (8) (1964) 1627-1639. 
349

350 [27] D. Veynante, T. Poinsot, J. Fluid Mech. 353 (1997) 83-114.

351 [28] Y.-C. Chen, R.W. Bilger, Combust. Flame 131 (2002) 400 - 435.

352 [29] D.B. Bradley, P.H. Gaskell, A. Sedaghat, X.J. Gu, Combust. Flame 135 $353 \quad$ (2003) 503-523.

354 [30] F.T.C. Yuen, Ö.L. Gülder, Proc. Combust. Inst. 32 (2009) 1747-1754.

355

356

357

358

[26] R.P. Lindstedt, E.M. Váos, Combust. Flame 116 (1999) 461-485.

[31] S. Gashi, J. Hult, K.W. Jenkins, N. Chakraborty, S. Cant, C.F. Kaminski, Proc. Combust. Inst. 30 (2005) 809-817.

[32] R. Sadanandan, P. Kutne, A. Steinberg, W. Meier, Flow. Turbul. Combust. 89 (2012) 275-294. 
Table 1: Measured turbulent flame brush $\left(\delta_{t}\right)$ and mean reaction layer (flame) $\left(\delta_{f}\right)$ thicknesses. The uncertainty in the turbulent flame brush thickness $\left(\Delta \delta_{t} / \delta_{t}\right)$ was obtained using the stochastic method of Goh [22]. All uncertainties in presented values illustrate the differences between upper and lower nozzles.

\begin{tabular}{ccccc}
\hline Fuel & $\phi$ & $\delta_{t}[\mathrm{~mm}]$ & $\delta_{f}[\mathrm{~mm}]$ & $\Delta \delta_{t} / \delta_{t}[\%]$ \\
\hline $\mathrm{C}_{5} \mathrm{H}_{10}$ & 0.75 & $8.13 \pm 0.47$ & $0.803 \pm 0.026$ & $0.44 \pm 0.21$ \\
$\mathrm{C}_{5} \mathrm{H}_{10}$ & 0.85 & $7.65 \pm 0.19$ & $0.751 \pm 0.026$ & $0.45 \pm 0.05$ \\
$\mathrm{JP}-10$ & 0.75 & $7.39 \pm 0.06$ & $0.829 \pm 0.052$ & $0.61 \pm 0.20$ \\
$\mathrm{JP}-10$ & 0.85 & $7.51 \pm 0.13$ & $0.829 \pm 0.000$ & $0.86 \pm 0.05$ \\
\hline
\end{tabular}

Table 2: Mean absolute uncertainties in flame front location $(\overline{|\Delta d|})$, orientation in degrees $(\overline{|\Delta \theta|})$ and curvature $(\overline{|\Delta h|})$. Comparison between fitted curves obtained using a SavitzkyGolay filter with $n_{f i t}=5,10$ and 15 .

\begin{tabular}{cccccc}
\hline Fuel & $\phi$ & $\begin{array}{c}n_{\text {fit }} \\
\text { range }\end{array}$ & $\begin{array}{c}\overline{|\Delta d|} \\
{[\mathrm{mm}]}\end{array}$ & $\begin{array}{c}\overline{|\Delta \theta|} \\
{\left[{ }^{\circ}\right]}\end{array}$ & $\begin{array}{c}\overline{|\Delta h|} \\
{\left[\mathrm{mm}^{-1}\right]}\end{array}$ \\
\hline $\mathrm{C}_{5} \mathrm{H}_{10}$ & 0.75 & $5 / 15$ & 0.00292 & 2.42 & 0.656 \\
$\mathrm{C}_{5} \mathrm{H}_{10}$ & 0.85 & $5 / 15$ & 0.00305 & 2.48 & 0.673 \\
$\mathrm{JP}_{10}$ & 0.75 & $5 / 15$ & 0.00298 & 2.55 & 0.658 \\
$\mathrm{JP}_{10} 10$ & 0.85 & $5 / 15$ & 0.00312 & 2.56 & 0.687 \\
$\mathrm{C}_{5} \mathrm{H}_{10}$ & 0.75 & $10 / 15$ & 0.00171 & 1.18 & 0.154 \\
$\mathrm{C}_{5} \mathrm{H}_{10}$ & 0.85 & $10 / 15$ & 0.00179 & 1.21 & 0.161 \\
$\mathrm{JP}_{10} 10$ & 0.75 & $10 / 15$ & 0.00177 & 1.28 & 0.157 \\
$\mathrm{JP}-10$ & 0.85 & $10 / 15$ & 0.00184 & 1.25 & 0.165 \\
\hline
\end{tabular}




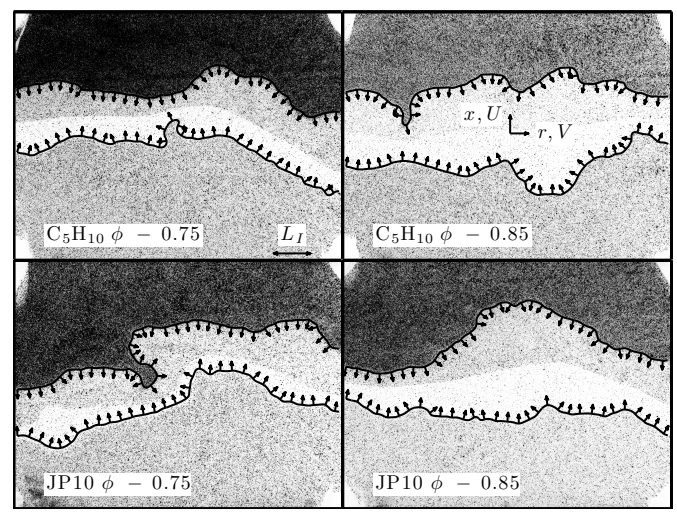

Figure 1: Sample PIV images for all cases, overlaid with detected flame fronts (lines). Mapped local flame normals (arrows) in direction of burnt products are also shown for sample points 50 pixels apart for clarity. The integral length scale $\left(L_{I}\right)$ is also shown.
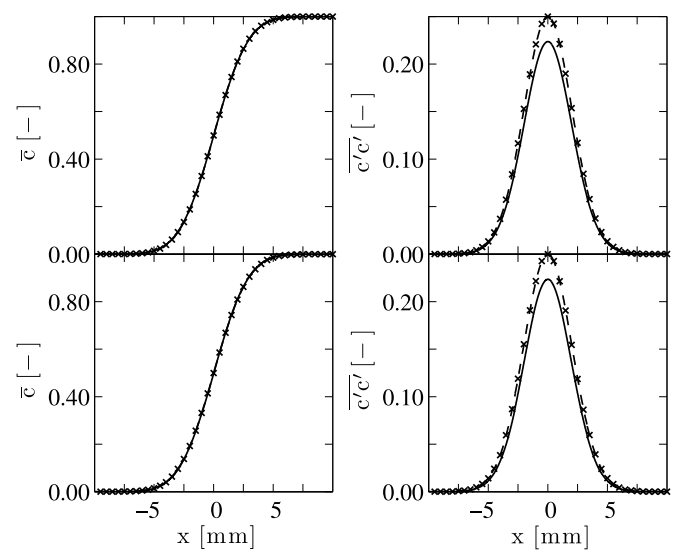

Figure 2: First (left) and second (right) moments of reaction progress variable statistics obtained taking into account finite thickness effects, using the method of Goh [22] for measured values of $\delta_{f}$ and $\delta_{t}$ for JP-10 at equivalence ratio of 0.75 . Top row - Actual values (solid) versus values obtained assuming zero interface (flame) thickness $\left(\delta_{f} \rightarrow 0\right)$ $(\times$ with dashed lines). Bottom row - Actual values (solid) versus values obtained with randomly displaced infinitesimally thin detected isocontours ( $\times$ with dashed lines). 


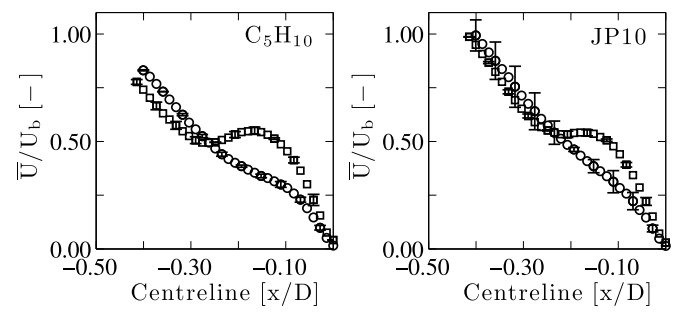

Figure 3: Mean axial velocity along axis of burner. Cyclopentane (left) and JP-10 (right) flames at equivalence ratios of $0.75(\bigcirc)$ and $0.85(\square)$.

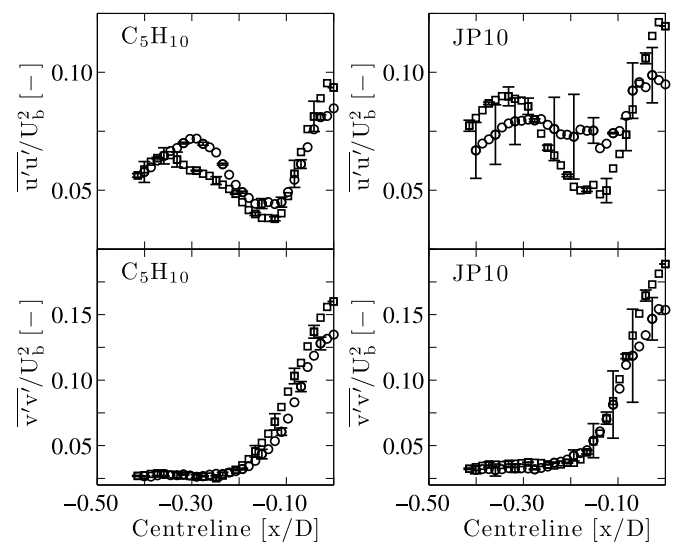

Figure 4: Mean axial (top) and radial (bottom) Reynolds stresses along axis of burner. Cyclopentane (left) and JP-10 (right) flames at equivalence ratios of $0.75(\bigcirc)$ and 0.85 $(\square)$. 

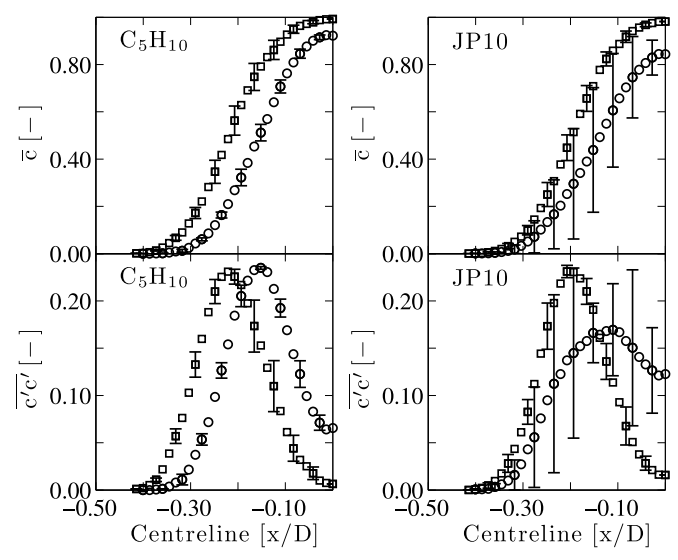

Figure 5: Mean first (top) and second (bottom) moments of reaction progress variable along axis of burner. Cyclopentane (left) and JP-10 (right) flames at equivalence ratios of $0.75(\bigcirc)$ and $0.85(\square)$.
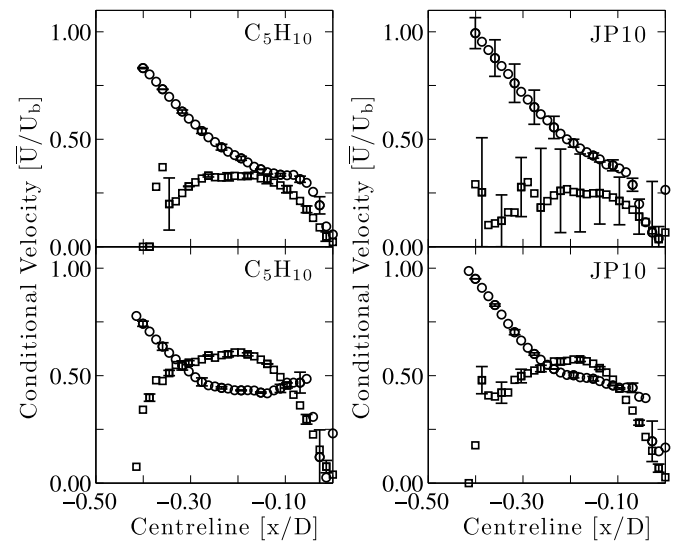

Figure 6: Mean axial reactant $\left(\bigcirc=\bar{U}_{r}\right)$ and product $\left(\square=\bar{U}_{p}\right)$ velocities along axis of burner. Cyclopentane (left) and JP-10 (right) flames at equivalence ratios of 0.75 (top) and 0.85 (bottom). 

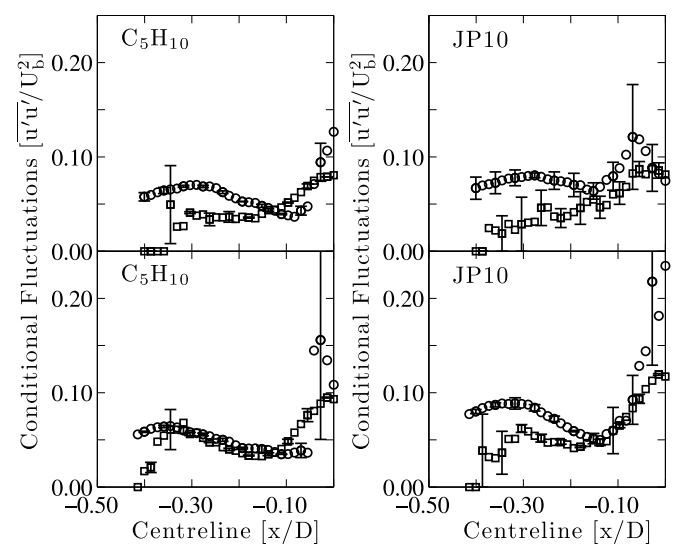

Figure 7: Mean axial reactant $\left(\bigcirc=\overline{u_{r}^{\prime} u_{r}^{\prime}}\right)$ and product $\left(\square=\overline{u_{p}^{\prime} u_{p}^{\prime}}\right)$ Reynolds stresses along axis of burner. Cyclopentane (left) and JP-10 (right) flames at equivalence ratios of 0.75 (top) and 0.85 (bottom).
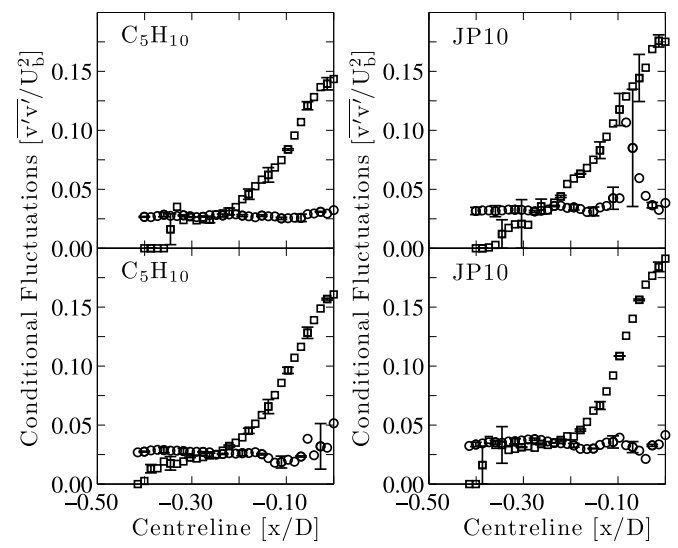

Figure 8: Mean radial reactant $\left(\bigcirc=\overline{v_{r}^{\prime} v_{r}^{\prime}}\right)$ and product $\left(\square=\overline{v_{p}^{\prime} v_{p}^{\prime}}\right)$ Reynolds stresses along axis of burner. Cyclopentane (left) and JP-10 (right) flames at equivalence ratios of 0.75 (top) and 0.85 (bottom). 


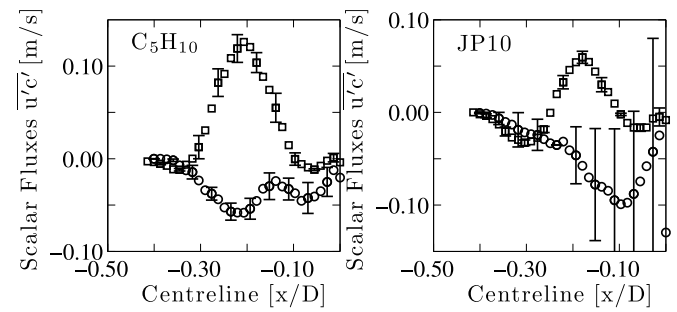

Figure 9: Mean axial scalar fluxes $\left(\overline{u^{\prime} c^{\prime}}\right)$ along axis of burner. Cyclopentane (left) and JP-10 (right) flames at equivalence ratios of $0.75(\bigcirc)$ and $0.85(\square)$.

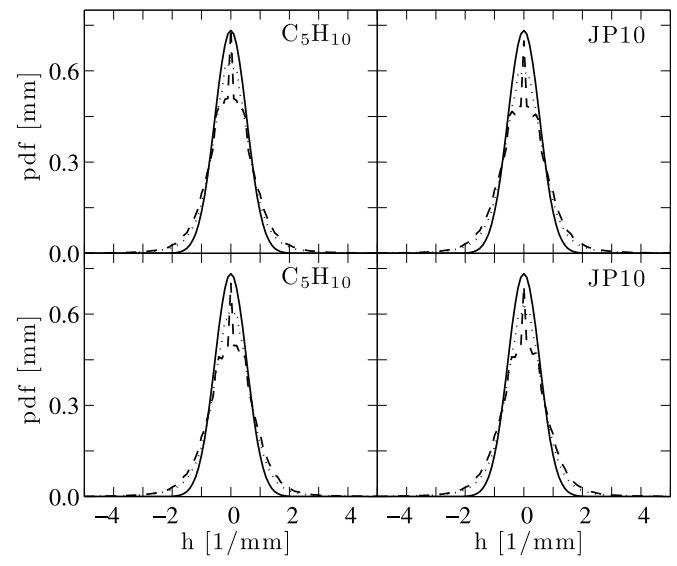

Figure 10: Curvature $p d f$ at resolution of $0.1[1 / \mathrm{mm}]$ for cyclopentane (left) and JP-10 (right) flames at equivalence ratios of 0.75 (top) and 0.85 (bottom). Savitzky-Golay filter with $n_{f i t}=10$ (dashed) and 15 (dotted). Empirical Gaussian fit by Bradley et al. [29] is also included for comparison (solid). 


\section{List of Figures}

1 Sample PIV images for all cases, overlaid with detected flame fronts (lines). Mapped local flame normals (arrows) in direction of burnt products are also shown for sample points 50 pixels apart for clarity. The integral length scale $\left(L_{I}\right)$ is also

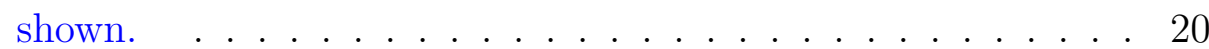

2 First (left) and second (right) moments of reaction progress variable statistics obtained taking into account finite thickness effects, using the method of Goh [22] for measured values of $\delta_{f}$ and $\delta_{t}$ for JP-10 at equivalence ratio of 0.75 . Top row - Actual values (solid) versus values obtained assuming zero interface (flame) thickness $\left(\delta_{f} \rightarrow 0\right)(\times$ with dashed lines). Bottom row - Actual values (solid) versus values obtained with randomly displaced infinitesimally thin detected isocontours (× with dashed lines). . . . . . . . . . . . . . . 20

3 Mean axial velocity along axis of burner. Cyclopentane (left) and JP-10 (right) flames at equivalence ratios of $0.75(\bigcirc)$ and $0.85(\square) . \ldots \ldots \ldots \ldots \ldots \ldots \ldots$

4 Mean axial (top) and radial (bottom) Reynolds stresses along axis of burner. Cyclopentane (left) and JP-10 (right) flames at equivalence ratios of $0.75(\bigcirc)$ and $0.85(\square)$. . . . . . . 21

5 Mean first (top) and second (bottom) moments of reaction progress variable along axis of burner. Cyclopentane (left) and JP-10 (right) flames at equivalence ratios of $0.75(\bigcirc)$ and $0.85(\square)$ 
6 Mean axial reactant $\left(\bigcirc=\bar{U}_{r}\right)$ and product $\left(\square=\bar{U}_{p}\right)$ velocities along axis of burner. Cyclopentane (left) and JP-10 (right) flames at equivalence ratios of 0.75 (top) and 0.85 (bottom). . 22

$7 \quad$ Mean axial reactant $\left(\bigcirc=\overline{u_{r}^{\prime} u_{r}^{\prime}}\right)$ and product $\left(\square=\overline{u_{p}^{\prime} u_{p}^{\prime}}\right)$ Reynolds stresses along axis of burner. Cyclopentane (left) and JP-10 (right) flames at equivalence ratios of 0.75 (top) and 0.85 (bottom). . . . . . . . . . . . . . . 23

$8 \quad$ Mean radial reactant $\left(\bigcirc=\overline{v_{r}^{\prime} v_{r}^{\prime}}\right)$ and product $\left(\square=\overline{v_{p}^{\prime} v_{p}^{\prime}}\right)$ Reynolds stresses along axis of burner. Cyclopentane (left) and JP-10 (right) flames at equivalence ratios of 0.75 (top) and 0.85 (bottom). . . . . . . . . . . . . . 23

9 Mean axial scalar fluxes $\left(\overline{u^{\prime} c^{\prime}}\right)$ along axis of burner. Cyclopentane (left) and JP-10 (right) flames at equivalence ratios of $0.75(\bigcirc)$ and $0.85(\square) . \ldots \ldots \ldots \ldots \ldots$

10 Curvature $p d f$ at resolution of $0.1[1 / \mathrm{mm}]$ for cyclopentane (left) and JP-10 (right) flames at equivalence ratios of 0.75 (top) and 0.85 (bottom). Savitzky-Golay filter with $n_{f i t}=10$ (dashed) and 15 (dotted). Empirical Gaussian fit by Bradley et al. [29] is also included for comparison (solid). . . . . . . . 24 